\title{
DEPRESIÓN Y CALIDAD DE VIDA RELACIONADA CON LA SALUD EN PACIENTES CON ARTROSIS: DIFERENCIAS DE GÉNERO
}

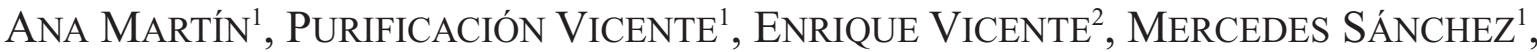 \\ PURIFICACIÓN GALINDO ${ }^{1}$ Y MANUEL MARTÍN ${ }^{3}$ \\ ${ }^{1}$ Departamento de Estadística, Universidad de Salamanca \\ 2 Centro de Salud Alfonso Sánchez Montero, Salamanca \\ ${ }^{3}$ Hospital Nuestra Señora de Sonsoles, Ávila
}

\begin{abstract}
Resumen: La artrosis es una enfermedad reumática frecuente que se asocia a un deterioro importante de la calidad de vida relacionada con la salud (CVRS). Sin embargo, el impacto de la artrosis no es igual en ambos sexos. El objetivo de este trabajo fue examinar el papel mediador de la depresión y del dolor severo en las diferencias de género en la CVRS de personas con artrosis. Se administraron dos cuestionarios, el EuroQol-5D y el Inventario de Depresión de Beck, a los 135 pacientes incluidos en el estudio. Se examinó, por separado, el efecto mediador de la depresión y del dolor severo en la relación entre género y CVRS, verificándose una mediación parcial en ambos casos. El estudio realizado muestra la conveniencia de que los médicos responsables de estos enfermos vigilen los síntomas de depresión, principalmente en las mujeres, con el fin de mejorarles la CVRS.
\end{abstract}

Palabras clave: Artrosis, depresión, calidad de vida relacionada con la salud, mediación.

\section{Depression and health-related quality of life in patients with osteoarthritis: Gender differences}

\begin{abstract}
Osteoarthritis is a common rheumatic disease, associated with an important deterioration in health-related quality of life (HRQL). However, arthritis impact is not the same in men and women. This study examined depression and severe pain as mediators between gender and HRQL in patients with arthritis. Two questionnaires, EuroQol-5D and the Beck Depression Inventory, were administrated to 135 patients included in the study. The effect of depression and severe pain as mediators in the relationship between gender and HRQL were examined separately, establishing a partial mediation in both cases. This study shows that physicians attending patients with arthritis, especially women, should control for symptoms of depression in order to improve HRQL.
\end{abstract}

Keywords: Osteoarthritis, depression, health-related quality of life, mediation.

\section{INTRODUCCIÓN}

La artrosis u osteoartritis es una enfermedad reumática frecuente que afecta a millones de personas en el mundo. Ocurre con más frecuencia en personas de edad media y ancianos, comprometiendo sobre todo las rodillas, las caderas, las articulaciones de los dedos y las regiones cervical y lumbar de la columna. La prevalencia

Recibido: 22-junio-2009; Aceptado: 6-octubre-2009

Correspondencia: Ana Martín Casado, Departamento de Estadística, Universidad de Salamanca, Espejo 2, 37007 Salamanca, España.

Correo electrónico: ammc@usal.es de la artrosis es mayor en las mujeres que en los hombres (Benito et al., 2008).

Existen diversos factores que aumentan el riesgo de padecer esta enfermedad: la obesidad, determinadas ocupaciones y actividades laborales, la herencia y la raza, y el excesivo ejercicio físico se cuentan entre ellos (Klippel, Stone, Crofford y White, 2008). La obesidad es uno de los factores más determinantes de deterioro de calidad de vida y discapacidad en pacientes con artrosis.

El principal síntoma de la artrosis es el dolor; suele aparecer cuando se exige un esfuerzo a la articulación enferma, bien sea de movimiento o de carga, y desaparece con el reposo. Al progre- 
sar la enfermedad el dolor aparece más precozmente y puede no cesar con el descanso.

La artrosis se asocia a un deterioro importante de áreas asociadas a la calidad de vida relacionada con la salud (CVRS) (deambulación, movimiento, cuidado corporal, comportamiento emocional, descanso nocturno, actividad doméstica y trabajo) (Carmona, Ballina, Gabriel, Laffon y EPISER Study Group, 2001), por lo que supone un impedimento para la vida cotidiana del paciente y un empeoramiento de su calidad de vida.

Sin embargo, el impacto de la artrosis sobre la CVRS no es igual es ambos sexos: las mujeres artrósicas aquejan más limitaciones en su trabajo, mayor angustia y dolor más severo que los hombres que padecen esta dolencia (Theis, Helmick y Hootman, 2007).

Teniendo en cuenta lo anterior, el objetivo de este trabajo fue examinar el papel mediador de la depresión y del dolor severo en las diferencias de género en la CVRS en personas diagnosticadas con artrosis.

\section{MÉTODO}

\section{Participantes}

El estudio, transversal y descriptivo, se realizó en el centro de Atención Primaria de Salamanca Alfonso Sánchez Montero durante los meses de abril a junio de 2007. Incluyó a los 135 pacientes con diagnóstico radiológico de artrosis, 44 hombres $(32,6 \%)$ y 91 mujeres $(67,4 \%)$, que acudieron a la consulta en esas fechas. Se explicó a cada paciente el objetivo del estudio, solicitando su participación. No hubo ningún paciente que rechazase participar en el mismo. Las edades de los participantes estaban comprendidas entre los 41 y los 94 años, con edad media de 68,23 (DT=10,53). En relación con otras características demográficas de la muestra, cabe destacar que: 1) en cuanto al nivel de estudios, sólo un $4,6 \%$ de sus integrantes tenía estudios secundarios y ninguno cursó estudios universitarios; 2) respecto a la ocupación, se observó un porcentaje muy alto de jubilados $(45,0 \%)$ y de amas de casa $(42,2 \%)$; y $3)$ en cuanto al estado civil, sólo 4 personas estaban solteras y 2 divorciadas, habiendo 82 casados $(60,7 \%)$ y 47 viudos $(34,8 \%)$. Sólo la ocupación se asoció con el género; sin embargo, esta variable no se relacionó significativamente con la CVRS.

\section{Instrumentos de evaluación}

EuroQol-5D (EQ-5D). Es un instrumento genérico de medición de la CVRS que puede utilizarse tanto en individuos relativamente sanos (población general) como en grupos de pacientes con diversas patologías (Herdman, Badia y Berra, 2001) que ha sido adaptado y validado para su uso en España (Badia, Roset, Montserrat, Herdman y Segura, 1999). Proporciona tres tipos de información: un perfil descriptivo de la calidad de vida del individuo en dimensiones, una puntuación de la calidad de vida global del individuo en una Escala Visual Analógica (EVA), y un valor que representa la preferencia del individuo por estar en un determinado estado de salud. Esta última información produce la obtención de una tarifa social de valores que, junto con los años de vida, forman un índice del resultado de las intervenciones sanitarias, el año de vida ajustado por la calidad. Dado el objetivo de este trabajo, sólo se administran las dos primeras partes de este cuestionario, esto es, el sistema descriptivo y la EVA. El sistema descriptivo consta de cinco dimensiones de salud: movilidad, cuidado personal, actividades cotidianas, dolor/malestar y ansiedad/depresión. Cada una de ellas tiene tres niveles de gravedad: 1 , sin problemas; 2 , algunos problemas o problemas moderados; y 3 , muchos problemas. Un estado de salud se define como la combinación de un nivel de problemas de cada dimensión. En total pueden formarse $243\left(=3^{5}\right)$ estados de salud distintos. El individuo señala el nivel de cada dimensión que le es aplicable en el momento de rellenar el cuestionario. La segunda parte del EQ-5D es una EVA vertical milimetrada de $20 \mathrm{~mm}$ que va desde 0 (peor estado de salud imaginable) hasta 100 (mejor estado de salud imaginable), donde el paciente debe marcar el punto que mejor refleje la valoración de su estado de salud global en el día en que cumplimenta el cuestionario. 
Beck Depression Inventory (BDI; Beck, Rush, Shaw y Emery, 1979). Consta de 21 ítems para evaluar la intensidad de la depresión, en cada uno de los cuales el sujeto tiene que elegir una frase entre un conjunto de cuatro opciones, ordenadas por su gravedad. Cada ítem se valora de 0 a 3 puntos en función de la opción escogida. La puntuación total de los 21 ítems varía, por tanto, de 0 a 63 puntos. Según la puntuación obtenida, el sujeto se clasifica en cuatro categorías (Beck y Steer, 1993): sin depresión (de 0 a 9 puntos), con depresión leve (de 10 a 16 puntos), con depresión moderada (de 17 a 29 puntos) y con depresión grave (de 30 a 63 puntos). En nuestro estudio, el BDI demostró buena fiabilidad (coeficiente alfa de Cronbach $=0,87$ ).

\section{Tratamiento estadístico de los datos}

Los métodos estadísticos empleados en nuestro estudio fueron: a) análisis de componentes principales (técnica de reducción de datos) para obtener puntuaciones de CVRS para cada paciente; b) coeficientes de correlación de Pearson para valorar la asociación entre dos variables cuantitativas; c) pruebas $F$ para comprobar si dos o más grupos difieren significativamente en sus puntuaciones de CVRS; d) análisis de regresión múltiple y de regresión logística para verificar si la depresión y el dolor median en la relación entre el género y la CVRS; y e) la prueba de $\chi^{2}$ para comprobar si dos variables cualitativas se asocian significativamente. Todos los cálculos fueron realizados usando el programa SPSS 15,0. De los 135 pacientes iniciales, uno no completó en su totalidad el EQ-5D y 3 no completaron el BDI. Estos pacientes quedaron excluidos en las pruebas que requerían ese tipo de información.

\section{RESULTADOS}

\section{Obtención de las puntuaciones de CVRS}

Dado que se observa que dos personas con el mismo perfil descriptivo pueden tener puntuaciones distintas en la EVA (por ejemplo, dos pacientes no presentaron problemas en ninguna de las cinco dimensiones y dieron una puntuación global en la EVA de 100 y 70, respectivamente), nos planteamos en primer lugar cómo obtener puntuaciones de CVRS que reflejen lo más objetivamente posible el estado de salud del individuo; esto es, puntuaciones que fueran iguales para pacientes con el mismo perfil del estado de salud. Abordamos la obtención de una medida tal de CVRS, empleando el análisis de componentes principales (ACP), técnica que permite resumir la información contenida en un conjunto de variables originales en un conjunto más pequeño de nuevas variables (factores) con una mínima pérdida de información. Así, se realizó un ACP con las cinco dimensiones del sistema descriptivo del EQ-5D, consiguiendo explicar más del 53\% de la información contenida en los datos con un único factor. Las puntuaciones factoriales obtenidas por cada sujeto en este único factor, transformadas en una escala de 0 a 100, fueron las puntuaciones de CVRS que se emplearon en análisis posteriores. Las puntuaciones dadas en la EVA por los pacientes sirvieron para comprobar la validez del constructo: la correlación entre las puntuaciones de CVRS obtenidas tras el ACP y las puntuaciones en la EVA fue $0,62(p<0,001)$ lo que indicó una asociación positiva significativa entre ambas.

La Tabla 1 muestra las puntuaciones de CVRS de los pacientes de la muestra en función de su género, su nivel de depresión y si tienen o no dolor acusado (puntuación $3 \mathrm{y}$ puntuaciones $1 \mathrm{y}$ 2, respectivamente, en la dimensión 4 del EQ-5D). En primer lugar, se compararon las puntuaciones de CVRS entre hombres y mujeres. Como puede observarse, las puntuaciones de los hombres y las mujeres difieren significativamente, mostrando que las mujeres con artrosis tienen peor CVRS que los hombres con esta dolencia (IC 95\% para la diferencia de medias de 6,4 a 21,8).

Asimismo, el análisis de la varianza realizado para comparar las puntuaciones de CVRS en los distintos niveles de depresión (sin depresión, con depresión leve, con depresión moderada y con depresión grave), muestra que estas puntuaciones difieren significativamente $(p<0$, 001). Las comparaciones por pares realizadas usando el ajuste de Bonferroni revelaron que las puntuaciones de CVRS de los pacientes sin de- 
presión y las de los pacientes con depresión leve no presentan diferencias significativas; asimismo, tampoco presentan diferencias significativas las puntuaciones de los pacientes con depresión moderada y las de los pacientes con depresión. Sin embargo, sí existían diferencias significativas entre las puntuaciones de CVRS de los pacientes sin depresión o con depresión leve y las de los pacientes con depresión moderada o con depresión grave. Por tanto, en lo que sigue consideraremos a los participantes en nuestro estudio clasificados en dos grupos atendiendo al nivel de depresión: el primero, integrado por los que no tienen depresión o la tienen leve; y el segundo, por los que tienen depresión moderada o grave.

También las puntuaciones de los pacientes sin dolor o con dolor moderado difieren significativamente de las puntuaciones de los pacientes con dolor severo (IC 95\% para la diferencia de medias de 33, 2 a 44,6), los cuales, como cabría esperar, tienen peor CVRS $(p<0,001)$. Examinamos a continuación si la depresión y el dolor severo actúan como mediadores en la relación entre género y CVRS, con el fin de esclarecer el proceso por el que el género afecta a la calidad de vida de las personas con artro-

Tabla 1. Puntuaciones de CVRS de los pacientes con artrosis $(\mathrm{n}=135)$

\begin{tabular}{lcccc}
\hline & $n$ & Media & $D T$ & $F$ \\
\hline Género & & & & $10,80^{* * *}$ \\
$\quad$ Hombres & 44 & 74,2 & 18,9 & \\
Mujeres & 90 & 60,2 & 25,1 & $14,26^{* * *}$ \\
Nivel de depresión & & & & \\
$\quad$ Sin depresión & 39 & 78,0 & 20,1 & \\
$\quad$ Con depresión leve & 42 & 69,7 & 19,7 & \\
$\quad$ Con depresión moderada & 41 & 50,9 & 22,7 & \\
$\quad$ Con depresión grave & 10 & 46,0 & 24,7 & \\
Dolor & & & & \\
$\quad$ Sin dolor o con dolor moderado & 95 & 76,1 & 17,2 & \\
$\quad$ Con mucho dolor & 39 & 37,2 & & \\
\hline$* * * p *, 1$ &
\end{tabular}

Tabla 2. Resumen de los análisis de regresión realizados para comprobar si la depresión media en la relación entre género y CVRS

\begin{tabular}{cccccc}
\hline & $R_{a}^{2}$ & $\hat{\beta}$ & $D T \hat{\beta}$ & $t$ & $p$ \\
\hline Modelo 1 & 0,287 & & & & \\
Género & & $-11,1$ & 3,8 & $-2,918$ & 0,004 \\
Modelo 2 & 0,421 & & & & \\
Género & & $-5,1$ & 3,7 & $-1,394$ & 0,166 \\
Depresión & & $-19,4$ & 3,5 & $-5,519$ & 0,000 \\
\hline & $\hat{\beta}$ & $D T \hat{\beta}$ & Wald & $p$ & Odds ratio \\
\hline Modelo 3 & & & & & \\
Género & 1,643 & 0,476 & 11,905 & 0,001 & 5,171 \\
\hline
\end{tabular}


Tabla 3. Resumen de los análisis de regresión realizados para comprobar si el dolor severo media en la relación entre género y CVRS

\begin{tabular}{lccccc}
\hline & $R_{a}^{2}$ & $\hat{\beta}$ & $D T \hat{\beta}$ & $t$ & $p$ \\
\hline $\begin{array}{l}\text { Modelo 1 } \\
\text { Género }\end{array}$ & 0,287 & & & & \\
Modelo 4 & & 3,8 & & \\
$\quad$ Género & 0,582 & & & & \\
$\quad$ Dolor severo & & $-2,9$ & 3,0 & $-0,966$ & 0,336 \\
\hline & $\hat{\beta}$ & $-33,4$ & 3,5 & $-9,567$ & 0,000 \\
\hline Modelo 5 & & WT $\hat{\beta}$ & & $p$ & Odds ratio \\
Género & 1,894 & 0,657 & 8,304 & 0,004 & 6,649 \\
\hline
\end{tabular}

sis. En este proceso, controlamos la edad y la articulación afectada por ser variables que influyen en la CVRS de los pacientes con artrosis.

\section{Mediación de la depresión en la relación entre género y CVRS}

Los criterios que deben cumplirse para establecer una mediación son tres (Baron y Kenny, 1986): (1) La variable independiente debe estar relacionada con la variable dependiente; (2) La variable independiente debe estar relacionada con la variable que actúa como mediadora; y (3) La variable mediadora debe estar relacionada con la variable dependiente, cuando se controla la variable independiente. Además, la relación entre la variable independiente y la dependiente debería reducirse a 0 (mediación completa) o disminuir (mediación parcial) cuando se controla la variable mediadora.

Para examinar el efecto mediador de la depresión $(0$, sin depresión o con depresión leve; 1, con depresión moderada o grave), ajustamos tres modelos de regresión (Tabla 2): El modelo 1 , evalúa el efecto del género $(0$, hombre; 1 , mujer) sobre las puntuaciones de CVRS; el modelo 2 , evalúa los efectos del género y de la depresión sobre la CVRS; y, finalmente, el modelo 3, que es un modelo de regresión logística, evalúa si el género se relaciona con la depresión. Tal y como se aprecia en la Figura 1, que muestra gráficamente los resultados obtenidos, se produce una mediación parcial de la depresión en la relación entre el género y la CVRS. El test de Sobel (Sobel, 1982) muestra que la mediación es estadísticamente significativa $(z=2,93$; $p=0,003)$. Usando la fórmula de Mackinnon y Dwyer (1993), el 54, 1\% del efecto del género sobre la CVRS está mediado por la depresión.

\section{Mediación del dolor severo en la relación entre género y CVRS}

A continuación, examinamos el efecto mediador del dolor severo $(0$, sin dolor o con dolor moderado; 1, con mucho dolor). Para ello, ajustamos tres modelos de regresión (Tabla 3): El modelo 1, ajustado anteriormente, evalúa el efecto del género sobre la CVRS; el modelo 4, evalúa los efectos del género y del dolor severo sobre la CVRS; y, finalmente, el modelo 5 evalúa si el género se relaciona con el dolor severo. Se produce una mediación parcial del dolor severo en la relación entre el género y la CVRS (Figura 2). El test de Sobel indica que la mediación es estadísticamente significativa $(z=3,13 ; p=0$, 002). El 73,7\% del efecto del género sobre la CVRS está mediado por el dolor severo.

\section{Asociación entre depresión y dolor severo}

Los dos mediadores estudiados (depresión y dolor) están asociados significativamente 


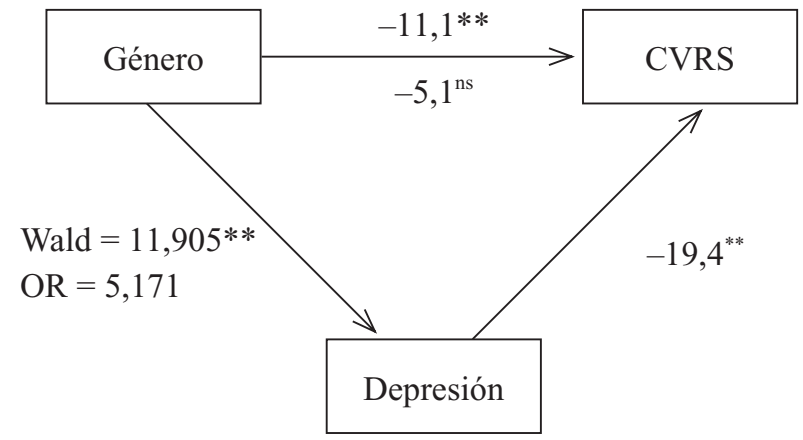

Figura 1. La depresión como mediador entre el género y la CVRS. OR=odds ratio; $* * \mathrm{p}<0,01 ;{ }^{\text {ns }}$ no significativo.

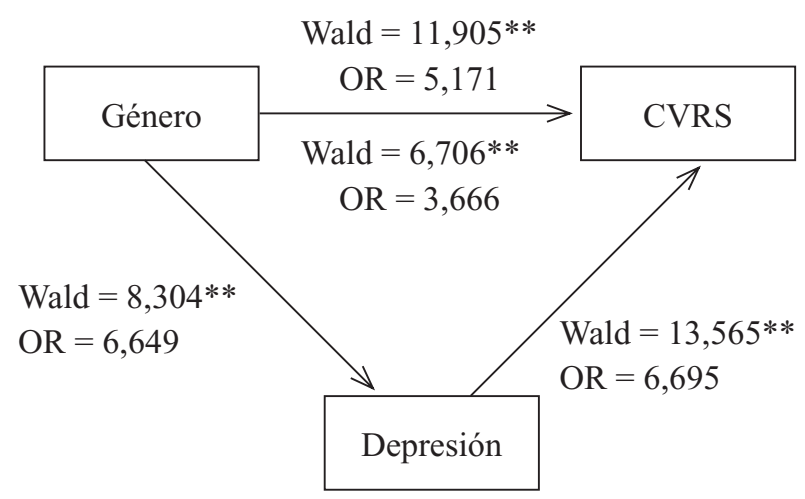

Figura 3. El dolor severo como mediador entre género y depresión. ${ }^{* *} \mathrm{p}<0,01 ; * \mathrm{p}<0,05$.

$\left(\chi^{2}=21,854, p=0,000\right)$. Ajustando los modelos de regresión logística pertinentes, se observa que el dolor puede mediar en la relación entre género y depresión (Figura 3) y/o que la depresión puede mediar en la relación entre género y dolor (Figura 4).

\section{DISCUSIÓN}

En este trabajo se estudió la relación entre género y CVRS en pacientes con artrosis, examinando la depresión y el dolor severo como posibles mediadores en esta relación. Diversos estudios (Theis, Helmick y Hootman, 2007; Rosemann, Laux y Szecsenyi, 2007; Theis, Murphy, Hootman, Helmick y Yelin, 2002) han mostrado que el impacto de la artrosis es mayor en las mujeres que en los hombres, lo que con-

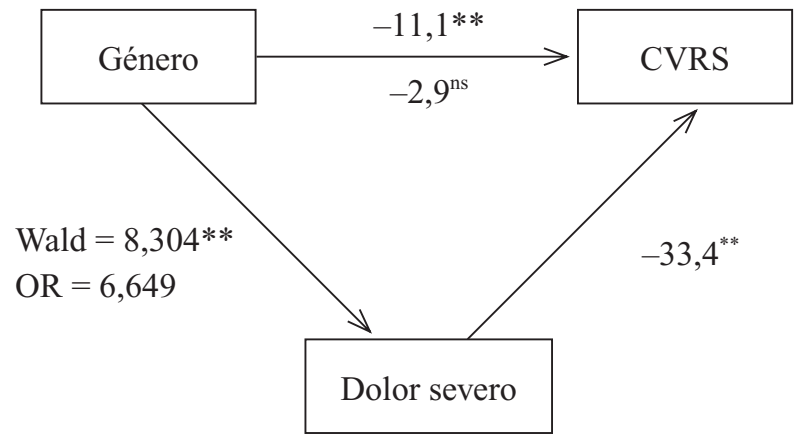

Figura 2. El dolor severo como mediador entre el género y la CVRS. ** $\mathrm{p}<0,01$; ${ }^{\text {ns }}$ no significativo.

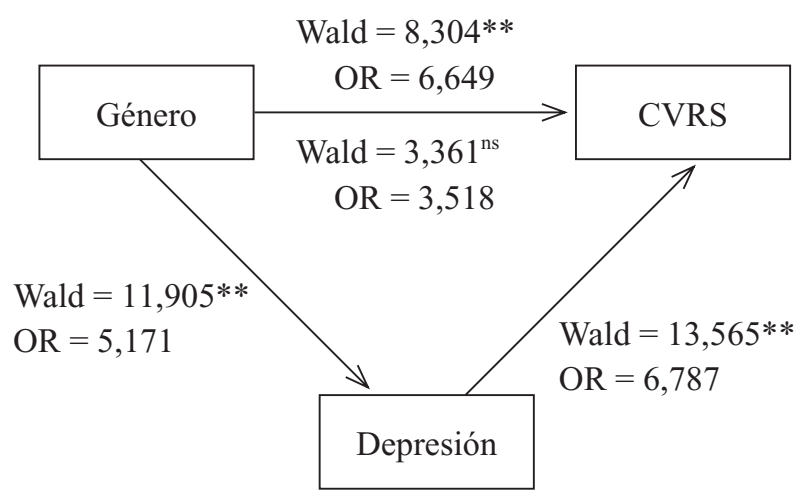

Figura 4. La depresión como mediador entre género y dolor severo. ${ }^{* *} \mathrm{p}<0,01 ;{ }^{\text {ns }}$ no significativo.

cuerda con nuestros datos. Por otra parte, las diferencias de género en la prevalencia de la depresión están bien documentadas (Coscollá, Caro, Calvo y López, 2008; Grigoradis y Robinson, 2007; Nolen-Hoeksema, Larson y Grayson, 1999), así como la diferencia en las manifestaciones depresivas según el género (Winkler et al., 2004; Boughton y Street, 2007). La depresión es dos veces más común en las mujeres que en los hombres, siendo también un problema más persistente en éstas. Las diferencias de género en depresión son máximas en las personas mayores de 34 años, en las que tienen hijos, en las casadas o divorciadas, en las de bajo nivel de estudios y en las no profesionales (Matud, Guerrero y Matías, 2006).

En nuestra muestra de pacientes con artrosis, se corroboró la mayor prevalencia de la depresión en las mujeres que en los hombres, obser- 
vándose una alta prevalencia en ambos géneros. Así, el 16,2\% de los hombres de la muestra presentaron depresión moderada o depresión grave mientras que en las mujeres este porcentaje fue del $48,9 \%$.

Otro hallazgo obtenido en nuestro trabajo que aparece también recogido en otros estudios (Rosemann, Geischen, Sauer, Laux y Szecsenyi, 2007; Katon, Lin y Kroenke, 2007) fue el de que la depresión concomitante agrava el impacto de la artrosis significativamente, así como el de otras enfermedades crónicas (Vinaccia et al., 2008). Un ensayo clínico realizado (Lin et al., 2003) demostró que cuando se trata la depresión de aquellas personas de edad avanzada con artropatía degenerativa, se consigue un alivio del dolor, una mayor movilidad y, consecuentemente, una mejora en su CVRS. Estos autores incluyeron en su estudio a 1801 personas deprimidas de $60 \mathrm{o}$ más años, de los que 1001 (56\%) presentaban también artrosis. Los participantes fueron asignados a dos grupos: a uno de ellos, se les administró un tratamiento con antidepresivos y fueron referidos a un especialista en salud mental; el otro grupo (grupo de intervención), recibió medicamentos para su depresión y un programa de seis a ocho sesiones de psicoterapia diseñado por el Centro de Atención Primaria. Al año, se observó que los pacientes del grupo de intervención fueron dos veces más propensos a presentar una reducción del $50 \%$ en sus síntomas depresivos, en comparación con los del otro grupo, refiriendo además que la artrosis les interfirió menos en sus actividades diarias. También aumentó la movilidad y se redujo significativamente la intensidad del dolor entre los participantes que recibieron antidepresivos y psicoterapia.

Por otra parte, en la muestra estudiada por nosotros también se observó que la tasa de prevalencia del dolor severo fue más alta en las mujeres que en los hombres $(38,9 \%$ frente a $9,1 \%$ ). Diversos estudios han mostrado que las mujeres tienen dolor más recurrente, más severo y más persistente que los hombres (International Association for the Study of Pain, 2008; Godfrey y Mackey, 2008; Tsai, 2007). Aunque muchas de las explicaciones sobre esta diferencia de género contemplan sólo aspectos relativos a mecanismos biológicos, resulta cada vez más evidente que los factores psicobiológicos son también importantes. Las mujeres tienen más probabilidades de experimentar depresión que los hombres y la depresión parece ser un factor de riesgo para las condiciones de dolor comunes.

El vínculo entre dolor y depresión ha sido estudiado por diversos autores. Así, Mcllvane, Schiaffino y Paget (2007) examinaron el daño funcional y el control personal como mediadores entre dolor y depresión en mujeres de mediana edad y ancianas con artrosis; Axford, Heron, Ross y Victor (2008) demostraron una relación compleja entre depresión, dolor, conocimiento de la enfermedad y capacidad física en pacientes con artrosis de rodilla; y Tsai (2007) sugirió que los efectos del género sobre el dolor son mediados por la depresión.

En los últimos años ha habido un debate notable en la literatura acerca de la relación causal entre dolor y depresión en artrósicos (Nicassio, 2007; Smith y Zautra, 2008). La naturaleza de nuestro estudio impide la comprensión de la relación temporal entre ambos conceptos, crucial para establecer una relación de causa-efecto. Sugerimos una relación recíproca, en la que la depresión aumentaría el dolor y viceversa.

Nuestro estudio presenta algunas limitaciones. Primera, la muestra utilizada fue una muestra obtenida por muestreo consecutivo y, por tanto, el resultado de su análisis puede estar sesgado de modos desconocidos. Segunda, el tamaño muestral fue pequeño lo que exige contrastar los resultados a mayor escala. Y tercera, la naturaleza transversal del estudio realizado impidió establecer relaciones de causalidad entre los dos mediadores examinados. Cabe destacar la necesidad de realizar en el futuro estudios de cohorte que permitan comprender mejor la asociación existente entre dolor y depresión.

A pesar de las limitaciones descritas, el estudio realizado por nosotros aclara el porqué las mujeres con artrosis tienen peor CVRS que los hombres con esta enfermedad y sugiere un manejo clínico diferente en ambos grupos. Asimismo pone de relieve la conveniencia de que los médicos responsables de estos enfermos vigilen los síntomas de depresión, principalmente en las mujeres, con el fin de mejorarles la CVRS. 


\section{REFERENCIAS}

Axford, J., Heron, C., Ross, F., Victor, C.R. (2008). Management of knee osteoarthritis in primary care: Pain and depression are the major obstacles. Journal of Psychosomatic Research, 64, 461-467.

Badia, X., Roset, M., Montserrat, S., Herdman, M., Segura, A. (1999). La version española del EuroQol: descripción y aplicaciones. Medicina Clínica, 112 (Supl 1), 79-86.

Baron, R.M., Kenny, D.A. (1986). The moderator-mediator variable distinction in social psychological research: Conceptual, strategic, and statistical considerations. Journal of Personality \& Social Psychology, 51, 1173-1182.

Beck, A.T., Rush, A.J., Shaw, B.F., Emery, G. (1979). Cognitive Therapy of Depression. New York: Guilford Press.

Beck, A.T., Steer, R.A. (1993). Beck Depression Inventory. Manual. San Antonio, TX: The Psychological Corporation.

Benito, P., Calvet, J., Lisbona, P., Martínez, J., Möller, I., Monfort, J., et al. (2008). Guía de buena práctica clínica en Geriatría: Artrosis. Madrid: Sociedad Española de Geriatría y Gerontología, Sociedad Española de Reumatología y Elsevier España.

Boughton, S., Street, H. (2007). Integrated review of the social and psychological gender differences in depression. Australian Psychologist, 42, 187-197.

Carmona, L., Ballina, J., Gabriel, R., Laffon, A., EPISER Study Group (2001). The burden of musculoskeletal diseases in the general population of Spain: results from a national survey. Annals of Rheumatic Diseases, 60, 1040-1045.

Coscollá, A., Caro, I., Calvo, I., López, B. (2008). Estudio psicológico de los adolescentes con enfermedades reumáticas: una primera aproximación. Revista de Psicopatología y Psicología Clínica, 13, 97-109.

Godfrey, J.R., Mackey, S. (2008). Toward optimal health: A discussion on sex, gender and pain. Journal of Womens Health, 17, 917-920.

Grigoriadis, S., Robinson, G.E. (2007). Gender issues in depression. Annals of Clinical Psychiatry, 9, 247-255.

Herdman, M., Badia, X., Berra, S. (2001). El EuroQol-5D: una alternativa sencilla para la medición de la calidad de vida relacionada con la salud en atención primaria. Atención Primaria, 28, 425-429.

International Association for the Study of Pain (IASP) (2008). Global year against pain in women. Journal of Pain \& Palliative Care Pharmacotherapy, 22, 90-91.

Katon, W., Lin, E.H.B., Kroenke, K. (2007). The association of depression and anxiety with medical symptom burden in patients with chronic medical illness. General Hospital Psychiatry, 29, 147-155.

Klippel, J.H., Stone, J.H., Crofford, L.J., White, P.H., editors (2008). Primer on the Rheumatic Diseases. Thirteenth Edition. New York: Springer.

Lin, E.H.B., Katon, W., von Korff, M., Tang, L., Williams, J.W. Jr, Kroenke, K., et al. (2003). Effect of improving depression care on pain and functional outcomes among older adults with arthritis. Journal of American Medical Association, 290, 2428-2439.

Mackinnon, D.P., Dwyer, J.H. (1993). Estimating mediated effects in prevention studies. Evaluation Review, 17, 144-158.

Matud, M.P., Guerrero, K., Matías, R.G. (2006). Relevancia de las variables sociodemográficas en las diferencias de género en depresión. International Journal of Clinical and Health Psychology, 6, 7-21.

McIlvane, J.M., Schiaffino, K.M., Paget, S.A. (2007). Age differences in the pain-depression link for women with osteoarthritis. Functional impairment and personal control as mediators. Womens Health Issues, 17, 44-51.

Nicassio, P.M. (2007). Understanding the role and significance of gender differences in pain and depression in Chinese elders with osteoarthritis. Pain, 130, 6-7.

Nolen-Hoeksema, S., Larson, J., Grayson, C. (1999). Explaining the gender differences in depressive symptoms. Journal of Personality \& Social Psychology, 77, 1061-1072.

Rosemann, T., Laux, G., Szecsenyi, J. (2007). Osteoarthritis: quality of life, comorbidities, medication and health service utilization assessed in a large sample of primary care patients. Journal of Orthopaedic Surgery, 2, 12-20.

Rosemann, T., Gensichen, J., Sauer, N., Laux, G., Szecsenyi, J. (2007). The impact of concomitant depression on quality of life and health service utilization in patients with osteoarthritis. Rheumatology International, 27, 859-863.

Smith, B.W., Zautra, A.J. (2008). The effects of anxiety and depression on weekly pain in women with arthritis. Pain, 138, 354-361.

Sobel, M.E. (1982). Asymptotic intervals for indirect effects in structural equations models. En: Leinhart S, editor. Sociological Methodology. San Francisco: Jossey-Bass, p. 290-312.

Theis, K.A., Helmick, C.G., Hootman, J.M. (2007). Arthritis burden and impact are greater among U.S. women than men: Intervention opportunities. Journal of Women Health, 16, 441-453.

Theis, K.A., Murphy, L., Hootman, J.M., Helmick, C.G., Yelin, E. (2007). Prevalence and correlates of arthritisattributable work limitation in the US population among persons ages 18-64: 2002 National Health Interview Survey Data. Arthritis and Rheumatism, 57, 355-363.

Tsai, Y.F. (2007). Gender differences in pain and depressive tendency among Chinese elders with knee osteoarthritis. Pain, 130, 188-194.

Vinaccia, S. et al. (2008). Calidad de vida relacionada con la salud en adultos diagnosticados con epilepsia. Revista de Psicopatología y Psicología Clínica, 13, 85-96.

Winkler, D., Pjrek, E., Heiden, A., Wieseger, N., Klein, N., Konstantinidis, A., et al. (2004). Gender differences in the psychopathology of depressed inpatients. European Archives of Psychiatry and Clinical Neuroscience, 54, 209-214. 